\title{
Nd-Zn (Neodymium-Zinc)
}

\author{
H. Okamoto
}

The Nd-Zn phase diagram in [Massalski2] was redrawn from [1972Mas].

Recently, this system was thermodynamically assessed by [2008Li], [2008Qi], and [2009Liu] independently. These assessments used the same experimental phase boundary data of [1972Mas] as the basis of their thermodynamic models. Accordingly the calculated phase diagrams were very similar except handling of the $\mathrm{NdZn}_{11}-\mathrm{NdZn}_{12}$ phase. According to [1972Mas], $\mathrm{NdZn}_{12}$ is the high-temperature modification of $\mathrm{NdZn}_{11}$, which has a solid solubility range of approximately 1 at.\%. [2008Li] and [2009Liu] assumed only $\mathrm{NdZn}_{11}$ exists as a line compound, whereas [2008Qi] assumed that $\mathrm{NdZn}_{11}$ undergoes a polymorphic transformation at about $550{ }^{\circ} \mathrm{C}$.

Figure 1 shows the $\mathrm{Nd}-\mathrm{Zn}$ phase diagram taken from [2008Li] except the $\mathrm{NdZn}_{11}-\mathrm{NdZn}_{12}$ phase area. In this phase diagram, both $\mathrm{NdZn}_{11}$ and $\mathrm{NdZn}_{12}$ are shown as line compounds, but the peritectoidal formation temperature of $\mathrm{NdZn}_{11}$ and the eutectoidal decomposition temperature of
$\mathrm{NdZn}_{12}$ are taken from [1972Mas]. Although this seems to be the most natural explanation of the existing experimental data, further confirmation is required.

\section{References}

1972Mas: J.T. Mason and P. Chiotti, The Nd-Zn Phase Diagram, Metall. Trans., 1972, 3, p 2851-2855

2008Li: H. Li, X. Su, Y.L. Liu, Z. Li, and X. Wang, Thermodynamic Assessment of the Nd-Zn System, J. Alloys Compd., 2008, 457, p 344-347

2008Qi: H. Qi, Z. Jin, L. Liu, and H. Liu, Thermodynamic Assessment of the Nd-Zn Binary System, J. Alloys Compd., 2008, 458, p 184-188

2009Liu: X.J. Liu, X. Chen, and C.P. Wang, Thermodynamic Assessment of the $\mathrm{Sm}-\mathrm{Zn}$ and $\mathrm{Nd}-\mathrm{Zn}$ Systems, J. Alloys Compd., 2009, 468, p 115-121

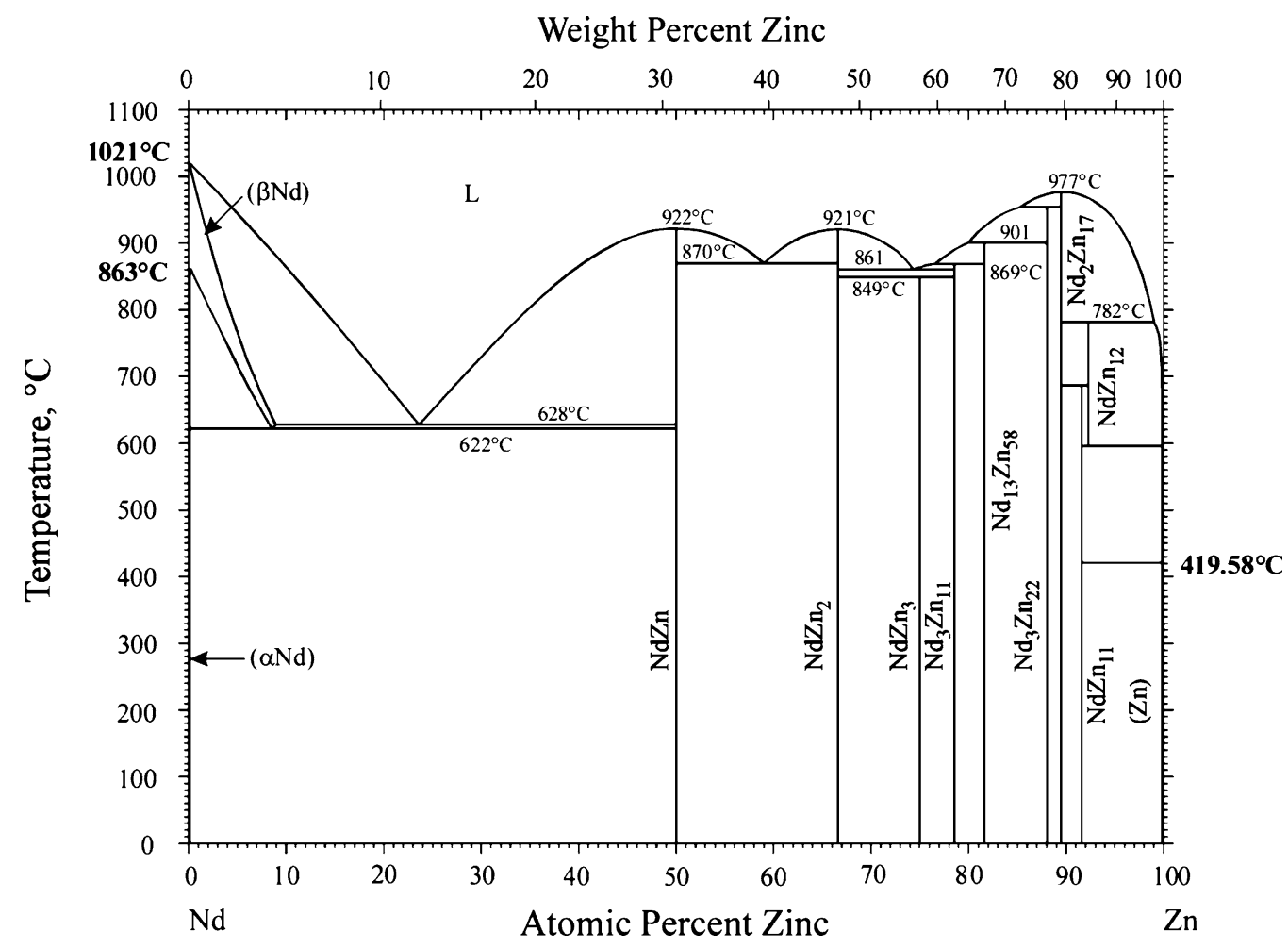

Fig. $1 \mathrm{Nd}-\mathrm{Zn}$ phase diagram 